\title{
Pulverização em alvos artificiais: avaliação com o uso do software conta-gotas
}

\author{
Spraying on artificial targets: evaluation with conta-gotas software
}

\author{
Marcelo Gonçalves Balan ${ }^{1}$ Otavio Jorge Grigoli Abi Saab ${ }^{2}$ \\ Inês Cristina de Batista Fonseca ${ }^{2}$ Cristhiane Gonzaga da Silva ${ }^{3}$ \\ Enio Hyroyuki Sasaki ${ }^{3}$
}

\section{- NOTA -}

\section{RESUMO}

A maioria dos métodos que avaliam as pulverizações agrícolas, além de subjetiva, é de difícil execução. Com o desenvolvimento da informática, há a possibilidade de as análises serem processadas em grande número e de forma não subjetiva. $O$ objetivo deste trabalho foi avaliar se o software Conta-Gotas pode ser utilizado para estudar o efeito do tamanho da gota, do vento e da orientação do bico na cobertura de uma pulverização em alvos artificiais. Na aplicação de cima para baixo, os resultados obtidos para as variáveis cobertura e número de gotas $\mathrm{cm}^{-2}$ podem ser considerados como esperados e mostram que, apesar de simples, o método tem sensibilidade para detectar diferenças. Considerando a orientação do bico, de cima para baixo e de baixo para cima, os resultados mostraram que a inversão provoca alterações no comportamento das gotas de pulverização em relação ao seu tamanho e efeito do vento. $O$ software conta-gotas é adequado para ser usado em avaliações semelhantes às realizadas neste trabalho.

Palavras-chave: deposição de gotas; cobertura; pontas de pulverização.

\section{ABSTRACT}

The majority of methods, which evaluates the pulverization in agriculture, besides being subjective it has

\begin{abstract}
difficult performance. With the development of the computer science, there is a possibility of processing analyses in great number and in a no subjective way. The objective of this work was to evaluate if the Conta-Gotas software can be used to study the effect of the drop size, the wind and the orientation of the spray nozzles in willing targets spraying coverage. From the top to the bottom application, the obtained results for the following variables as covering and droplets $\mathrm{cm}^{-2}$ can be considered as expected and they show that, in spite of being simple, the method has sensibility to detect differences. Considering the top to the bottom and vice-versa spray nozzles orientation the results show how the simple inversion provokes alterations in the behavior of the pulverization drops in relation to its size and effect of the wind. The conta-gotas software is appropriate to be used in similar evaluations like the ones done in this work.
\end{abstract}

Keywords: droplet deposition; coverage; spray nozzles.

O uso da pulverização na agricultura depende não somente de produtos de ação comprovada, mas também da tecnologia desenvolvida para sua aplicação. A pulverização fica ainda condicionada ao momento de sua realização e a influência dos fatores meteorológicos e biológicos. A

${ }^{1}$ Bolsista do Conselho Nacional de Pesquisa e Desenvolvimento Científico e Tecnológico(CNPq), Mestrando em Agronomia, Universidade Estadual de Londrina, autor para correspondência, Rua Irati, 66, 86060-540, Londrina, PR, Brasil. Email:balan@uel.br.

${ }^{2}$ Departamento de Agronomia, Centro de Ciências Agrárias, Universidade Estadual de Londrina, Londrina, PR, Brasil.

${ }^{3}$ Discente do curso de Agronomia, Universidade Estadual de Londrina, Londrina, PR, Brasil. 
eficiência da pulverização é afetada pela forma, tamanho e posição do alvo, densidade, diâmetro e velocidade de gota e pela velocidade e direção do fluxo. ABI SAAB (1996) destaca que não necessariamente gotas menores promoverão melhores coberturas e deposições, dependendo, entre outros fatores, do efeito do vento e da orientação do bico. Segundo STEDEN (1992), a importância do tamanho das gotas cresce em função do aumento da dificuldade de alcance do alvo. Os fatores que influenciam as características da deriva, segundo SATOW et al. (1993), são, além do tamanho das gotas, velocidade, turbulência e direção do vento. O volume de aplicação, distância do alvo, pressão, velocidade e a energia cinética das partículas pulverizadas também influenciam diretamente a deriva. Ventos superiores a $10 \mathrm{~km} \mathrm{~h}^{-1}$ são altamente problemáticos à aplicação de defensivos porque favorecem o desvio da trajetória das partículas pulverizadas (MATTHEWS \& THORNHILL, 1994). Esses ventos podem jogar as gotas sobre o aplicador e, dependendo da posição em que se estiver aplicando, levá-las para áreas adjacentes. Os diâmetros das gotas formadas são diferentes nos diversos equipamentos para pulverização, apesar da alta tecnologia empregada. $\mathrm{O}$ ideal é que se consiga uma maior homogeneidade no tamanho das gotas, sendo que o bico de pulverização é o elemento que mais influencia na qualidade do tratamento desejado (BLANCA, 1999). Os métodos utilizados para avaliar as aplicações exigem condições encontradas apenas em laboratórios. O desenvolvimento de métodos mais simples e confiáveis é um desafio antigo. Basear-se apenas em avaliações visuais, mesmo que usando papel sensível à água, por exemplo, não é considerado adequado. Com o desenvolvimento da informática, é possível analisar amostras em número suficiente, e de maneira não subjetiva, permitindo conclusões mais confiáveis. Microcomputadores, scanners e softwares estão disponíveis em praticamente todos os lugares, o que facilita sobremaneira a possibilidade de realizar avaliações das aplicações que antes não seriam possíveis. O objetivo deste trabalho foi avaliar se o software Conta-Gotas (CANTERI et al., 2001) pode ser utilizado para estudar o efeito do tamanho da gota, do vento e da orientação do bico na cobertura de pulverizações em alvos artificiais.

$\mathrm{O}$ experimento foi realizado em ambiente fechado, com temperatura de $20^{\circ} \mathrm{C}$ e umidade relativa do ar de $70 \%$, condições obtidas com o uso de um aparelho de ar condicionado. Os alvos artificiais utilizados foram tiras de papel cartolina branca, com dimensões de 10 x 4cm, dispostos horizontalmente.
As pulverizações foram efetuadas utilizando-se equipamento costal equipado com válvula reguladora de pressão. A calda aplicada foi uma mistura de água com corante preto para tinta látex na proporção de $0,5 \%$. O experimento foi separado em duas partes. Na primeira, o bico foi orientado de cima para baixo e, na segunda, de baixo para cima, em relação à posição do alvo. Para os tratamentos, utilizaram-se dois tipos de pontas de pulverização tipo leque, 110-LD-02 e 110-SF-02, gerando gotas denominadas, neste trabalho, de maiores (GMa) e menores (GMn), respectivamente. As aplicações foram realizadas sempre a $0,50 \mathrm{~m}$ do alvo, pressão de trabalho de $200 \mathrm{kPa}$, vazão de $0,68 \mathrm{~L} \mathrm{~min}^{-1} \mathrm{e}$ velocidade de deslocamento de $6 \mathrm{~km} \mathrm{~h}^{-1}$. Cada aplicação foi repetida na presença de vento com velocidade média de $10 \mathrm{~km} \mathrm{~h}^{-1}$, gerado por ventiladores dispostos de maneira a promover um efeito lateral. Considerou-se a primeira aplicação como sem efeito do vento (SV) e a segunda como com o efeito do vento $(\mathrm{CV})$. Os ventiladores, apesar de não recomendados para simular vento em experimentos deste tipo, foram utilizados devido à impossibilidade de executar o experimento em condições ideais. A temperatura, a umidade relativa do ar e a velocidade do vento foram monitoradas por um termohigroanemômetro. Os alvos foram digitalizados com 300 dpi de resolução em preto e branco em um Scanner, gerando um arquivo de imagem cujas cores originais (preta e branca) foram transformadas pelo software Corel Draw $10^{\hat{a}}$ para azul e amarelo, respectivamente, para serem processadas pelo software Conta-Gotas (CANTERI et al., 2001). As faces dos alvos foram denominadas superior (S) e inferior (I). As variáveis analisadas foram: cobertura (área percentual coberta pela aplicação) e número de gotas por centímetro quadrado. As duas partes do experimento foram analisadas em esquema fatorial $2 \mathrm{x}$ $2 \times 2$ (tamanho de gota $\mathrm{x}$ face do alvo $\mathrm{x}$ vento), com 10 repetições. As análises estatísticas foram feitas através da análise de variância complementada com o teste de Tukey para comparação de médias, em nível de probabilidade de erro de $5 \%$.

Os resultados das duas partes do experimento, onde apareceram diferenças significativas, estão apresentados na tabela $1 . \mathrm{Na}$ primeira parte, com orientação do bico de cima para baixo em relação ao alvo, observou-se a presença de gotas, independente do tamanho ou do efeito do vento, apenas na face superior dos alvos. Esse fato pode ser atribuído ao tipo de ponta utilizada, de jato plano ou leque, que geralmente é indicada para pulverizações com gotas maiores e que, naturalmente, tem menor capacidade de cobertura da face do alvo contrária ao sentido da aplicação. 
Tabela 1 - Análise de variância da porcentagem de cobertura e número de gotas por centímetro quadrado, com as respectivas médias, dos fatores tamanho da gota, vento, e da interação entre eles, da face superior dos alvos, quando com orientação de pulverização de cima para baixo e da face superior e inferior dos alvos, em pulverização de baixo para cima.

Orientação de pulverização de cima para baixo - face inferior

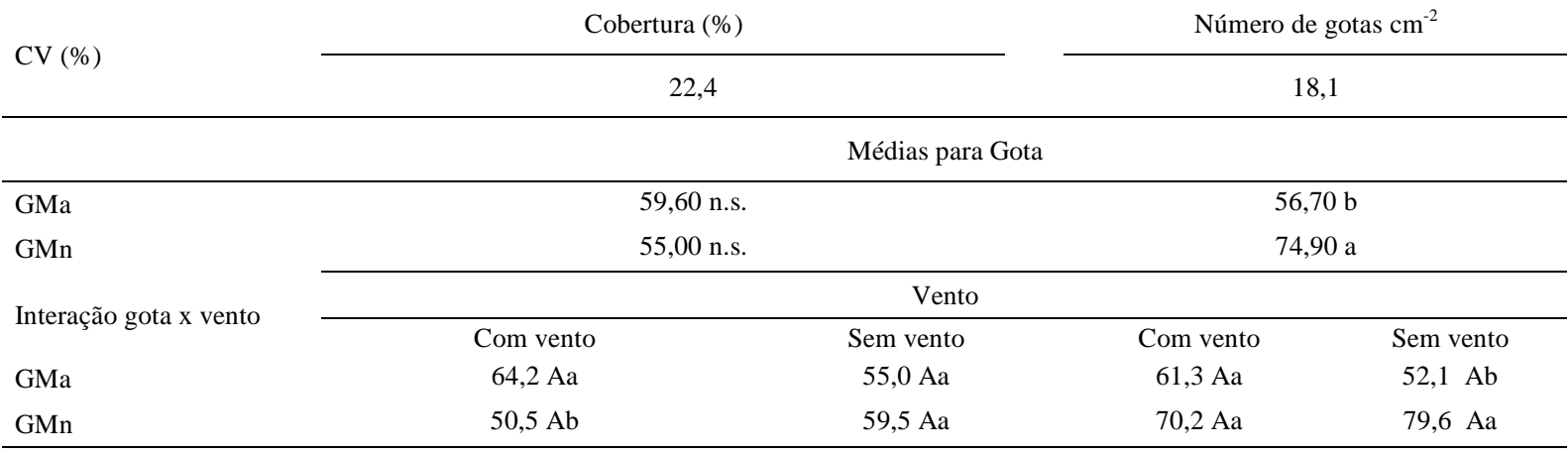

Orientação de pulverização de baixo para cima Cobertura (\%) Número de gotas $\mathrm{cm}^{-2}$

$\mathrm{CV}(\%)$

41,8

36,7

\begin{tabular}{|c|c|c|c|c|c|}
\hline & \multicolumn{5}{|c|}{ Médias para gota } \\
\hline GMa & \multicolumn{3}{|c|}{$15,525 \mathrm{a}$} & \multicolumn{2}{|c|}{21,950 n.s. } \\
\hline GMn & \multicolumn{3}{|c|}{$12,475 \mathrm{~b}$} & \multicolumn{2}{|c|}{22,850 n.s. } \\
\hline & \multicolumn{5}{|c|}{ Médias para vento } \\
\hline $\mathrm{CV}$ & \multicolumn{3}{|c|}{$13,250 \mathrm{~ns}$} & \multicolumn{2}{|c|}{$19,625 \mathrm{~b}$} \\
\hline \multirow[t]{2}{*}{ SV } & \multicolumn{3}{|c|}{$14,750 \mathrm{~ns}$} & \multicolumn{2}{|c|}{$25,150 \mathrm{a}$} \\
\hline & \multicolumn{5}{|c|}{ Médias para face } \\
\hline$S$ & \multicolumn{3}{|c|}{$05,550 \mathrm{~b}$} & \multicolumn{2}{|c|}{$13,200 \mathrm{~b}$} \\
\hline I & \multicolumn{3}{|c|}{$22,450 \mathrm{a}$} & \multicolumn{2}{|c|}{$31,575 \mathrm{a}$} \\
\hline \multirow{2}{*}{ Interação gota $\mathrm{x}$ vento } & \multicolumn{5}{|c|}{ Vento } \\
\hline & \multicolumn{2}{|r|}{$\mathrm{CV}$} & SV & $\mathrm{CV}$ & SV \\
\hline GMa & \multicolumn{2}{|r|}{$17,00 \mathrm{Aa}$} & $14,05 \mathrm{Aa}$ & $22,75 \mathrm{Aa}$ & $21,15 \mathrm{Ab}$ \\
\hline GMn & \multicolumn{2}{|r|}{$09,50 \mathrm{Bb}$} & $15,45 \mathrm{Aa}$ & $16,50 \mathrm{Bb}$ & $29,15 \mathrm{Aa}$ \\
\hline \multirow{2}{*}{ Interação gota $x$ face } & \multicolumn{5}{|c|}{ Face } \\
\hline & \multicolumn{2}{|r|}{ S } & I & $S$ & I \\
\hline GMa & \multicolumn{2}{|r|}{$4,95 \mathrm{Ba}$} & $26,10 \mathrm{Aa}$ & $11,55 \mathrm{~ns}$ & $32,35 \mathrm{~ns}$ \\
\hline GMn & \multicolumn{2}{|r|}{$6,15 \mathrm{Ba}$} & $18,80 \mathrm{Ab}$ & $14,85 \mathrm{~ns}$ & $30,80 \mathrm{~ns}$ \\
\hline \multirow{2}{*}{ Interação vento $\mathrm{x}$ face } & & & Face & & \\
\hline & & $\mathrm{S}$ & I & $\mathrm{S}$ & I \\
\hline $\mathrm{CV}$ & & $4,45 \mathrm{~ns}$ & $22,05 \mathrm{~ns}$ & $09,40 \mathrm{~ns}$ & $29,85 \mathrm{~ns}$ \\
\hline SV & & $6,65 \mathrm{~ns}$ & $22,85 \mathrm{~ns}$ & $17,00 \mathrm{~ns}$ & $33,30 \mathrm{~ns}$ \\
\hline & & & e $\mathrm{x}$ vento & & \\
\hline 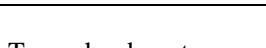 & $\Gamma=$ & & & & \\
\hline Tamanho de gota & Face & $\mathrm{CV}$ & SV & $\mathrm{CV}$ & SV \\
\hline GMa & S & $4,60 \mathrm{Ab}$ & $5,30 \mathrm{Ab}$ & $10,40 \mathrm{~ns}$ & $12,70 \mathrm{~ns}$ \\
\hline GMa & I & $29,40 \mathrm{Aa}$ & $22,80 \mathrm{Ba}$ & $35,10 \mathrm{~ns}$ & $29,60 \mathrm{~ns}$ \\
\hline GMn & S & $4,30 \mathrm{Ab}$ & $8,00 \mathrm{Ab}$ & $08,40 \mathrm{~ns}$ & $21,30 \mathrm{~ns}$ \\
\hline GMn & I & $14,70 \mathrm{Ba}$ & $22,90 \mathrm{Aa}$ & $24,60 \mathrm{~ns}$ & $37,00 \mathrm{~ns}$ \\
\hline
\end{tabular}

GMa e GMn: gotas maiores e menores, respectivamente; CV e SV: com vento e sem vento, respectivamente. S e I: face superior e inferior, respectivamente. As médias seguidas de mesma letra, maiúsculas nas linhas e minúsculas nas colunas, não diferem entre si pelo teste de Tukey no nível de $5 \%$ de probabilidade. n.s. indica que não houve significância. 
No entanto, a completa ausência das gotas na face inferior dos alvos sugere que o método avaliado pode não ter a sensibilidade para detectar gotas menores. Para os resultados da cobertura, houve diferença significativa apenas na interação Gota $x$ Vento, com as gotas maiores propiciando melhor cobertura, sob condição de vento, em comparação às menores. Portanto, o uso de gotas maiores, sob condições de vento, apresentou menor potencial de ocorrência de deriva e ventos com velocidades de $10 \mathrm{~km} \mathrm{~h}^{-1}$ favoreceram o desvio da trajetória das partículas pulverizadas, concordando com MATTHEWS \& THORNHILL (1994). O número de gotas por centímetro quadrado, na interação Gota $x$ Vento, apresentou diferença significativa na pulverização efetuada com gotas menores, sendo superior na condição de ausência de vento. No entanto, com presença de vento, esta diferença não foi significativa. Esse resultado também corrobora o entendimento geral que as gotas menores são mais sujeitas ao desvio do alvo, devido à deriva (SARKER et al., 1997). A aplicação, de cima para baixo, representa a maioria dos casos de pulverização com equipamentos de barras, predominante na produção de grãos. Os resultados obtidos para as variáveis de cobertura e número de gotas $\mathrm{cm}^{-2}$ podem ser considerados como esperados.

Com orientação do bico de baixo para cima, segunda parte do experimento, observou-se a presença de gotas nas duas faces dos alvos, independente do tamanho da gota ou do efeito do vento. Ocorreu interação para Gota x Face x Vento para a cobertura dos alvos. A cobertura foi significativamente maior na face inferior, independente do vento e do tamanho das gotas. $\mathrm{Na}$ face superior, a cobertura foi significativamente maior com a ausência de vento para as gotas menores, conforme o esperado. No entanto, para as gotas maiores, a presença de vento resultou em uma cobertura significativamente maior na face superior dos alvos. O número de gotas $\mathrm{cm}^{-2}$ foi significativamente maior na face inferior dos alvos, que recebeu diretamente a pulverização, havendo somente interação Gota x Vento. Para as gotas maiores, o fator vento não causou diferença significativa, mas, para as menores, a presença de vento diminui significativamente o número de gotas $\mathrm{cm}^{-2}$. Na comparação do tamanho das gotas, o vento provocou aumento significativo nas gotas maiores, ocorrendo o inverso no caso da ausência do mesmo. Esse fato é esperado, pois, para um mesmo volume de aplicação, um DMV menor determina um número de gotas maior. No entanto, com vento, as gotas menores estarão mais sujeitas aos efeitos da deriva. Segundo ABI SAAB (1996), em aplicações de baixo para cima, as gotas maiores podem proporcionar melhor cobertura. $\mathrm{O}$ autor também atribuiu esse comportamento à quantidade de movimento adquirido pelas gotas (momento linear), função da velocidade e da massa da gota, que leva uma gota de massa maior a uma maior distância, quando arremessada de baixo para cima. Esta maior massa faz com que a gota sofra menor influência de outras forças como, por exemplo, o vento.

Os resultados mostram que a inversão da orientação do bico provoca alterações no comportamento das gotas de pulverização em relação ao seu tamanho e efeito do vento. O software ContaGotas apresentou desempenho satisfatório e pode ser utilizado em avaliações semelhantes. Entretanto, devese aprofundar os estudos visando ao estabelecimento do número adequado de amostras.

\section{REFERÊNCIAS}

ABI SAAB, O.J.G. Avaliação de um sistema de aplicação de defensivos utilizado em videiras no Município de Londrina/PR. 1996. 65f. Dissertação (Mestrado em Agronomia/ Energia na Agricultura) - Faculdade de Ciências Agronômicas, Universidade Estadual Paulista.

BLANCA, A.L. Maquinaria agrícola: constituición, funcionamiento, regulación y cuidados. 3.ed. Madrid: Ministério da Agricultura, Pesca e Alimentação, 1999. $361 \mathrm{p}$.

CANTERI, M.G. et al. Conta-gotas: sistema para análise de eficiência de pulverização. In: CONGRESSO PAULISTA DE FITOPATOlOGiA, 24., 2001, Piracicaba, SP. Summa Phytopathologica. Jaboticabal : Grupo Paulista de Fitopatologia, 2001. v.27, p.136.

MATTheWs, G.A.; THORNHILl, E.W. Pesticide application equipment for use in agriculture. Rome : FAO, 1994. 171p.

SATOW, T. et al. Influence of droplet size of spray on drift characteristics. Res Bull Obihiro Univ, v.18, p.97-104, 1993.

SARKER, K.U. et al. Effects of liquid properties on the potential for spray drift from flat-fan hydraulic nozzles. In: BRIGHTON CROP PROTECTION CONFERENCE, 1997, Brighton. Proceedings... Surrey : The British Crop Protection Council, 1997. p.555-560.

STEDEN, C. Untersuchungen zum einflub der tropfengröbe auf die belagsbildung und die biologische wirksamkeit gegen Oidium tuckeri Berk. an reben. 1992. 118f. Inaugural Dissertation (zur Erlangung des Doktorgrades Justus-Liebig-Universität Gieben) - Justus-LiebigUniversität Gieben. 